\title{
Exploring Potential Synergism Between Darunavir/ Cobicistat and Ribavirin for the Treatment of Covid-19 Patients: A Retrospective Comparison Study
}

\author{
Reem Elajez ${ }^{1 *}$, Oraib Abdallah ${ }^{1}$, Shaikha Alnaimi ${ }^{1}$, Eman Elmekaty ${ }^{1}$, Safae Abuyousef ${ }^{1}$, Eiman Abdelfattah \\ Arafa $^{1}$, Raja Barazi ${ }^{1}$, Rola Ghasoub ${ }^{1}$, Mohammed Abu khattab ${ }^{2}$ and Moza Al Hail ${ }^{1}$ \\ ${ }^{1}$ Department of Pharmacy, Hamad Medical Corporation, Qatar \\ ${ }^{2}$ Infectious disease section, Department of Medicine, Hamad Medical Corporation, Qatar
}

\begin{abstract}
Objective: Exploring potential synergistic effect of darunavir/cobicistat (DRV/COBI) plus ribavirin (RBV) in COVID-19 treatment.

Methods: Retrospective, observational comparison study conducted in Qatar. Adult patients with confirmed positive SARSCoV-2 who received either DRV/COBI or DRV/COBI+RBV were included. Primary outcome was time to viral clearance, while secondary outcomes. include clinical improvement (resolution of fever/tachypnea), development of acute respiratory distress syndromes (ARDS) and 28-days all-cause mortality, and incidence of adverse reactions.

Results: Sixty-four patients were included (41 received DRV/COBI and 23 received DRV/COBI+ RBV). Mean age of patients was $48.4 \pm 14.6$ years with predominant male gender (58/64; 90.6\%). Median time from starting therapy till viral clearance was 11 days in DRV/COBI+RBV vs. 16 days in DRV/COBI groups $(P=0.295)$. At day-28, viral clearance was observed in 82.6\% (19/23) and 73.2\% $(30 / 41)$ in DRV/COBI+RBV and DRV/COBI groups, respectively $(P=0.392)$. Patients receiving DRV/COBI+RBV did not achieve faster resolution of fever or tachypnea compared to those receiving DRV/COBI. Three patients in DRV/COBI+RBV group developed ARDS, while none in DRV/COBI group. Frequency of hemoglobin drop of $\geq 2 \mathrm{~g} / \mathrm{dl}$ was significantly higher in DRV/COBI+RBV group than in DRV/COBI group (34.8\% vs. 7.9\%, $P=0.008$, respectively).
\end{abstract}

Conclusion: Use of darunavir/cobicistat plus ribavirin was not associated with faster viral clearance or better clinical outcomes.

KEYWORDS: SARS-CoV-2; COVID-19; Ribavirin; Darunavir/cobicistat

ABBREVATIONS: ARDS: Acute Respiratory Distress Syndromes; DRV: Darunavir; COBI: Cobicistat; SARS-CoV: Severe Acute Respiratory Syndrome Coronavirus; CPE: Cytopathic Effect; PCR: Polymerase Chain Reaction; ICU: Intensive Care Unit; ECMO: Extracorporeal Membrane Oxygenation; IQR: Interquartile Range

\section{INTRODUCTION}

The novel coronavirus disease 2019 (COVID-19), caused by severe acute respiratory syndrome coronavirus 2 (SARS-CoV-2) was first identified in Wuhan-China in December 2019 as the cause of serial pneumonia cases [1]. In March 2020, the World Health
Organization (WHO) announced COVID-19 as a pandemic due to the dramatic increase in cases worldwide. As of August $12^{\text {th }}, 2020$, more than 20 million cases have been confirmed worldwide with 0.7 million deaths [2]. This urges the need for a better understanding of the disease and the possible treatment approaches.
Quick Response Code:

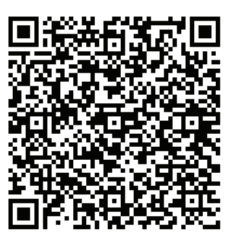

Address for correspondence: Reem Elajez, Department of Pharmacy, Hamad General Hospital, Hamad Medical Corporation, Qatar

Received: March 06, $2021 \quad$ Published: March 15, 2021

How to cite this article: Elajez R, Abdallah O, Alnaimi S, Elmekaty E, Abuyousef S, Abdelfattah E, Barazi R, Ghasoub R, Abu-Khattab M, Al-Hail M. Exploring Potential Synergism Between Darunavir/Cobicistat and Ribavirin for the Treatment of Covid-19 Patients: A Retrospective Comparison Study. 2021- 3(2) OAJBS.ID.000262. DOI: $10.38125 /$ OAJBS.000262 
Clinically, the spectrum of COVID-19 ranges from self-limiting respiratory tract illness to severe progressive pneumonia, acute respiratory distress syndrome (ARDS), multi-organ failure, and death [3-4]. The phylogenic analysis and full-genome sequencing of SARS-CoV-2 indicates that it is in the same subgenus as the severe acute respiratory syndrome coronavirus (SARS-CoV) [5] and hence similar clinical course and presentation. With SARS, the progression of the disease is mainly noted after the initial viral replicative phase [6]. This implies that for the treatment of SARS (and possibly COVID-19), a potential strategy would be to reduce the peak viral load at the earlier stages to prevent disease complication. This can possibly be achieved using an antiviral agent with a known inhibitory effect [7]. Multiple antiviral agents were previously studied as potential treatments for coronavirus infection; however, antiviral therapy with demonstrated efficacy is still limited. The most studied agents are remdesivir, protease inhibitors (lopinavir, darunavir), ribavirin, and favipiravir [8].

In vitro antiviral susceptibility testing showed that protease inhibitors (lopinavir, darunavir) and ribavirin had weak in vitro inhibitory effect when used separately for SARS [9]. Although the cytopathic effect (CPE) of SARS-CoV was inhibited by lopinavir at $4 \mu \mathrm{g} / \mathrm{mL}$ and by ribavirin at $50 \mu \mathrm{g} / \mathrm{mL}$, CPE inhibition was achieved down to a concentration of $1 \mu \mathrm{g} / \mathrm{mL}$ for lopinavir plus $6.25 \mu \mathrm{g} / \mathrm{mL}$ for ribavirin using checkerboard assay for synergy [9]. A previous study attempted to translate the in vitro synergism of lopinavir and ribavirin into clinical outcomes. The investigators reported that SARS-infected patients who received the combination therapy had significantly lower risk of adverse clinical outcomes (ARDS or death) compared to those who received monotherapy $(2.4 \%$ vs. $28.8 \%, P<0.001$ ) [9]. This observed benefit could be due to the synergistic effect between ribavirin and lopinavir.

Few studies investigated the potential role of protease inhibitors in COVID-19 patients [10-12]. In an open-label randomized controlled trial, lopinavir/ritonavir did not show any additional benefit compared to the standard care in COVID-19 patients [12]. Another open label randomized controlled trial concluded that five days of darunavir/cobicistat did not increase the proportion of negative viral conversion compared to standard of care alone [13]. However, these studies did not investigate the potential synergism of ribavirin in combination with lopinavir/ritonavir or darunavir/ cobicistat due to its potential synergism with them.

As clinical evidence of COVID-19 treatment options is still limited, initiatives to find the best treatment option are highly warranted. Based on available literature and the potential synergism of combination antivirals, we aim to compare the outcome of (darunavir/cobicistat) versus (darunavir/cobicistat plus ribavirin) for the treatment of COVID-19 patients. Up to our best knowledge, there is no previous study that compared the safety and efficacy of (darunavir/cobicistat) versus (darunavir/cobicistat plus ribavirin) in the management of COVID-19 pneumonia.

\section{METHODOLOGY}

\section{Study Design and Population}

We conducted a retrospective, observational chart review study that looked at COVID-19 positive patients who were treated in any COVID-19 facilities under Hamad Medical Corporation (HMC) in Qatar. Inclusion criteria were adults ( $\geq 18$ years), with a confirmed positive SARS-CoV-2 from respiratory tract specimen and had received either Darunavir/Cobicistat (DRV/COBI) or Darunavir/ Cobicistat with Ribavirn (DRV/COBI +RBV) as part of the local protocol followed in our institution for COVID-19 pneumonia between $29^{\text {th }}$ February to $29^{\text {th }}$ April 2020 . Exclusion criteria were receiving the study treatments for less than five days, and receiving interferon concurrently with ribavirin (as the focus of this study was to explore the potential synergism effect between ribavirin and darunavir/cobicistat, receiving interferon may augment the ribavirin effect and falsely impact the study outcomes).

SARS-CoV-2 was diagnosed by polymerase chain reaction (PCR) assays TaqPath COVID- 19 Combo Kit (Thermo Fisher Scientific, Waltham, Massachusetts) or Cobas SARS-CoV-2 Test (Roche Diagnostics, Rotkreuz, Switzerland). Darunavir/cobicistat was given at dose of 1 tablet ( $800 \mathrm{mg}$ darunavir/ $150 \mathrm{mg}$ cobicistat) once daily, and ribavirin orally as $2.4 \mathrm{~g}$ loading dose followed by $1.2 \mathrm{~g}$ twice daily (with dose adjustment in case of renal impairment). As the COVID-19 situation was fluid, management protocol of such patients was frequently changed based on the rapidly changing and emerging evidence. Thus, till 23/03/2020 treatment of COVID-19 pneumonia (based on local protocol in Qatar) consisted of both darunavir/cobicistat AND ribavirin (DRV/COBI + RBV group). Later, the updated version of local treatment protocol recommended darunavir/cobicistat for COVID-19 pneumonia without the need of adding ribavirin (DRV/COBI group).

Ethical approval for this study was obtained from HMC Medical research center (MRC-01-20- 394). All patients met inclusion and exclusion criteria were included, since sample size could not be calculated as this is the first study to compare the safety and efficacy of DRV/COBI vs. DRV/COBI + RBV in COVID-19 patients.

\section{Outcome Measures and Data Collection}

The primary outcome was time to viral clearance (viral clearance is defined as two consecutive negative COVID-19 PCR samples). Secondary outcomes were: (1) viral clearance by day $(7,14,21)$ and overall at day 28 (2) development of Acute Respiratory Distress Syndromes (ARDS) and all-cause mortality at day 28 (3) clinical deterioration (defined as intensive care unit (ICU) admission, intubation, vasopressor use, prone positioning, or extracorporeal membrane oxygenation (ECMO)), (4) length of hospital stay (5) clinical improvement (defined as resolution of fever and tachypnea), and (6) premature discontinuation of study treatment due to adverse drug reactions. Only ARDS and clinical deterioration that occurred $\geq 2$ days from starting the drug were counted as outcome of the study therapy. Demographic data (age, gender, comorbidities...), virology results, laboratory data, radiology findings, and concomitant COVID medications were collected. Clinical data including presenting symptoms, vital signs, signs of clinical deterioration were also collected. All outcomes were assessed by 28 days from starting study treatment.

As the combination of $\mathrm{DRV} / \mathrm{COBI}+\mathrm{RBV}$ is believed to be maximally effective during viral replication stage (viremia), the outcomes of patients who started study treatment within 7 days of symptoms onset (early initiation) were compared to those who started the treatment after 7 days from the onset of symptoms (late initiation) as subgroup analyses.

\section{Data Analysis}

Descriptive statistics were used to summarize demographics and all other characteristics of the patients. Categorical data were expressed by frequency (percentage), while continuous values expressed as mean \pm SD for normally distributed data or as median with interquartile range (IQR) for skewed data. The Shapiro-Wilk 
test was used for normality testing. Continuous data were examined with the Mann-Whitney $\mathrm{U}$ or independent t-test as appropriate while for categorical data we used the chi-square or Fischer's exact tests as appropriate. Variables with statistical significance in the univariate analysis were included in the multivariate analysis.

Outcomes were assessed using Cox proportional hazards model to identify the independent factors for viral clearance at day 28 and ARDS.

The time to viral clearance was represented by Kaplan-Meier plot and compared with a log-rank test. $P$-value of $<0.05$ was considered statistically significant. All statistical analyses were done using statistical package SPSS, version 25.

\section{RESULTS}

\section{Participants}

A total of 64 patients met our inclusion and exclusion criteria, where 41 patients received DRV/COBI and 23 patients received combination of DRV/COBI + RBV. The mean age was $48.4 \pm 14.6$ years $(47.59 \pm 15.6$ vs. $49.87 \pm 12.9 ; P=0.554$ for $D R V / C O B I$ and $\mathrm{DRV} / \mathrm{COBI}+\mathrm{RBV}$ groups respectively) with predominantly male gender (58/64; 90.6\%). less than one-quarter had 3 or more existing comorbidities (Table 1). Median duration to start the study medications was 7.5 (5-10) days after onset of symptoms and 3 (14) days from first positive RT-PCR for SARS-COv-2. Most patients did not need oxygen supplement in the first 24 hours from starting study treatment.

Table 1: Demographics and baseline characteristics.

\begin{tabular}{|c|c|c|c|}
\hline & $\begin{array}{c}\text { DRV/COBI } \\
\mathrm{N}=41\end{array}$ & $\begin{array}{c}\mathrm{DRV} / \mathrm{COBI}+\mathrm{RBV} \\
\mathrm{N}=\mathbf{2 3}\end{array}$ & $P$-value \\
\hline Age $[$ Mean $\pm S D]$ & $47.59 \pm 15.6$ & $49.87 \pm 12.9$ & 0.554 \\
\hline Male gender (\%) & $36(87.8)$ & $22(95.7)$ & 0.301 \\
\hline \multicolumn{4}{|l|}{ Ethnicity (\%) } \\
\hline Arab & $16(39)$ & $8(34.8)$ & 0.737 \\
\hline White/Caucasian (European descent) & $1(2.4)$ & $1(4.3)$ & 0.674 \\
\hline South Asian (Indian subcontinent) & $20(48.8)$ & $14(60.9)$ & 0.352 \\
\hline Southeast and West Asian (Chinese descent) & $2(4.9)$ & $0(0)$ & 0.282 \\
\hline Other & $2(4.9)$ & $0(0)$ & 0.282 \\
\hline Weight in kg [median (IQR)] & $76(68-91)$ & $80(68-90)$ & 0.839 \\
\hline Obesity (BMI >30) (\%) & $12(29.3)$ & $7(30.4)$ & 0.922 \\
\hline \multicolumn{4}{|l|}{ Baseline Co-existing Conditions (\%) } \\
\hline Myocardial Infarction & $2(4.9)$ & $2(8.7)$ & 0.545 \\
\hline Congestive Heart Failure & $1(2.4)$ & $0(0)$ & 0.45 \\
\hline Hypertension & $8(19.5)$ & $10(43.5)$ & $0.041^{*}$ \\
\hline COPD & $2(4.9)$ & $0(0)$ & 0.282 \\
\hline Asthma & $2(4.9)$ & $0(0)$ & 0.282 \\
\hline Diabetes Mellitus & $12(29.3)$ & $10(43.5)$ & 0.251 \\
\hline Chronic Kidney Disease & $5(12.2)$ & $2(8.7)$ & 0.667 \\
\hline Liver Disease & $0(0)$ & $1(4.3)$ & 0.178 \\
\hline Solid Tumor & $1(2.4)$ & $0(0)$ & 0.45 \\
\hline \multicolumn{4}{|l|}{ Smoking Status } \\
\hline Current smoker & $0(0)$ & $3(13)$ & $0.018^{*}$ \\
\hline Former smoker & $1(2.4)$ & $0(0)$ & 0.45 \\
\hline Never smoked & $37(90.2)$ & $17(73.9)$ & 0.084 \\
\hline Not documented & $3(7.3)$ & $3(13)$ & 0.451 \\
\hline Charlson score [median (IQR)] & $1(0-2)$ & $1(0-3)$ & 0.378 \\
\hline Number of comorbidities & & & 0.466 \\
\hline 0 & $20(48.8)$ & $8(34.8)$ & \\
\hline $1-2$ & $12(29.3)$ & $10(43.5)$ & \\
\hline$\geq 3$ & $9(22)$ & $5(21.7)$ & \\
\hline \multicolumn{4}{|l|}{$\begin{array}{l}\text { Baseline condition (within } 24 \text { h of therapy } \\
\text { initiation) }\end{array}$} \\
\hline Fever $\left(\geq 38^{\circ} \mathrm{C}\right)$ & $19(46.3)$ & 17(73.9) & $0.033^{*}$ \\
\hline Tachypnea (>24 breath/min) & $11(26.8)$ & $10(43.5)$ & 0.173 \\
\hline Oxygen supplement required & $12(29.3)$ & $11(47.8)$ & 0.325 \\
\hline
\end{tabular}




\begin{tabular}{|c|c|c|c|}
\hline \multicolumn{4}{|l|}{ Chest X-ray } \\
\hline Unilateral finding & $16(39)$ & $10(43.5)$ & 0.728 \\
\hline Bilateral finding & $25(61)$ & $13(56.5)$ & 0.728 \\
\hline CRUB-65 score & & & 0.464 \\
\hline $0-1$ & $36(87.8)$ & $18(78.3)$ & \\
\hline 2 & $3(7.3)$ & $4(17.4)$ & \\
\hline $3-5$ & $2(4.9)$ & $1(4.3)$ & \\
\hline \multicolumn{4}{|l|}{ Laboratory [median (IQR)] } \\
\hline WBC & $5.6(5-8.3)$ & $5.9(4.5-7.3)$ & 0.181 \\
\hline Lymphocyte count & $1.3(0.9-1.8)$ & $1(0.7-1.6)$ & 0.157 \\
\hline Platelet & $204(174-248)$ & $228(170-266)$ & 0.619 \\
\hline Creatinine & $83.5(77-96)$ & $80.5(75-94)$ & 0.866 \\
\hline ALT & $30(21-37)$ & $28(21-40)$ & 0.234 \\
\hline AST & $36(26-50)$ & $35(26-50)$ & 0.896 \\
\hline LDH & $252(190-283)$ & $404(365-449)$ & $0.029^{*}$ \\
\hline \multicolumn{4}{|l|}{ Other COVID Medications (\%) } \\
\hline Hydroxychloroquine/chloroquine & $40(97.6)$ & $22(95.7)$ & 0.674 \\
\hline Azithromycin & $38(92.7)$ & $20(87)$ & 0.451 \\
\hline \multicolumn{4}{|l|}{ Duration of Study Treatment [Median (IQR)] } \\
\hline Darunavir/Cobicistat & $7(5-13)$ & $13(11-14)$ & $0.001^{*}$ \\
\hline Ribavirin & NA & $10(7-11)$ & NA \\
\hline Time from symptoms [Mean \pm SD] & $7.9 \pm 3.5$ & $6.6 \pm 2.6$ & 0.144 \\
\hline $\begin{array}{l}\text { Duration from positive till initiate therapy [median } \\
\text { (IQR)] }\end{array}$ & $3(2-5)$ & $2(1-3)$ & 0.1 \\
\hline
\end{tabular}

Note: *Indicate statistically significant (P-value <0.05), BMI: Body Mass Index; COPD: Chronic Obstructive Pulmonary Disease; WBC: White Blood Cell; ALT: alanine transaminase; AST: aspartate transaminase; LDH: Lactate Dehydrogenase; NA: Not Applicable.

\section{Measured Outcomes}

Administration of $\mathrm{DRV} / \mathrm{COBI}+\mathrm{RBV}$ was not found to be associated with faster virological clearance (Figure 1). The median duration from starting the study treatment until a confirmed negative RT-PCR was 11 days and 16 days in the DRV/COBI + RBV and DRV/COBI groups, respectively $(P=0.295)$. The proportion of viral clearance at day 7 was higher in the DRV/COBI+ RBV group
$26.1 \%(6 / 23)$ compared to DRV/COBI group 17.1\% (7/41), though it was not statistically significant $(P=0.39)$. Similarly, there was no significant difference in viral clearance proportion at day 14 and 21 between DRV/COBI + RBV group vs. DRV/COBI group (Table 2 ). By the end of follow-up (day 28), viral clearance was achieved in $82.6 \%$ and $73.2 \%$ in DRV/COBI + RBV and DRV/COBI groups, respectively $(P=0.392)$.

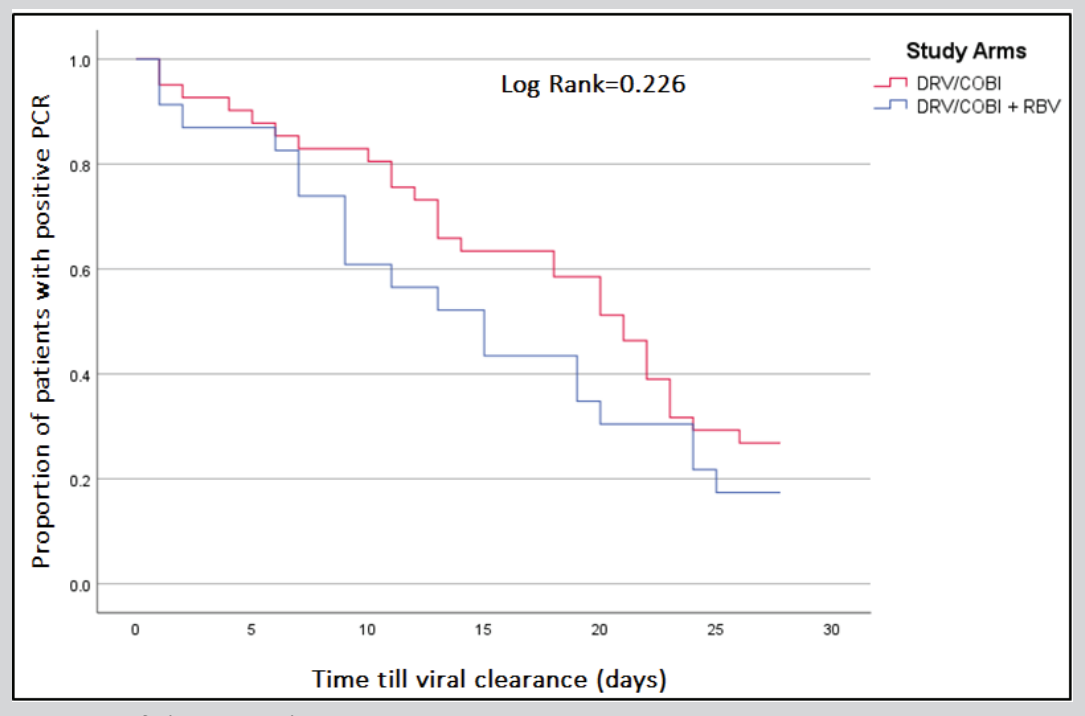

Figure 1: Kaplan-Meier curve of time to viral clearance. 
Table 2: Study outcomes.

\begin{tabular}{|c|c|c|c|}
\hline & $\begin{array}{c}\text { DRV/COBI } \\
\mathrm{N}=41\end{array}$ & $\begin{array}{c}\mathrm{DRV} / \mathrm{COBI}+\mathrm{RBV} \\
\mathrm{N}=\mathbf{2 3}\end{array}$ & $P$-value \\
\hline \multicolumn{4}{|l|}{ Viral clearance $(\%)$} \\
\hline$\leq 7$ days & $7(17.1)$ & $6(26.1)$ & 0.39 \\
\hline 8-14 days & $8(19.5)$ & $5(21.7)$ & 0.832 \\
\hline 15-21 days & $7(17.1)$ & $5(21.7)$ & 0.646 \\
\hline Overall (day 28) & $30(73.2)$ & $19(82.6)$ & 0.392 \\
\hline $\begin{array}{l}\text { Duration from therapy till clearance [median } \\
\text { [IQR)] }\end{array}$ & $16(9-22)$ & $11(7-19)$ & 0.295 \\
\hline \multicolumn{4}{|l|}{ Time to resolution of symptoms (Mean $\pm \mathrm{SD})$} \\
\hline Fever & $6.13 \pm 3.7$ & $7.53 \pm 3.9$ & 0.215 \\
\hline Tachypnea & $3.94 \pm 2.9$ & $10.31 \pm 12.8$ & 0.063 \\
\hline \multicolumn{4}{|l|}{ Achievement of symptoms resolution (\%) ${ }^{\#}$} \\
\hline Fever & $18(94.7)$ & $17(100)$ & 0.658 \\
\hline Tachypnea & $11(100)$ & $9(90)$ & 0.277 \\
\hline \multicolumn{4}{|l|}{ Highest Oxygen requirement (\%) } \\
\hline Room air & $22(53.7)$ & $8(34.8)$ & 0.147 \\
\hline Nasal cannula/Mask & $19(46.3)$ & $6(26.1)$ & 0.111 \\
\hline NIV & $0(0)$ & $0(0)$ & NA \\
\hline Mechanical Ventilation & $0(0)$ & $3(13)$ & $0.018^{*}$ \\
\hline Duration of oxygen requirement (Mean \pm SD) & $7.74 \pm 5.5$ & $11.13 \pm 8.1$ & 0.158 \\
\hline ICU admission (\%) & $0(0)$ & $4(17.4)$ & $0.006^{*}$ \\
\hline Length of ICU admission (Mean \pm SD) & NA & $10 \pm 8.6$ & NA \\
\hline Vasopressor therapy (\%) & $0(0)$ & $2(8.7)$ & 0.055 \\
\hline Intubation (\%) & $0(0)$ & $3(13)$ & $0.018^{*}$ \\
\hline Length of Intubation (Mean \pm SD) & NA & $4.3 \pm 2.5$ & NA \\
\hline ARDS (\%) & $0(0)$ & $3(13)$ & $0.018^{*}$ \\
\hline Time from therapy initiation till ARDS & NA & $4 \pm 1$ & NA \\
\hline Prone positioning (\%) & $0(0)$ & $2(8.7)$ & 0.055 \\
\hline ECMO (\%) & $0(0)$ & $0(0)$ & NA \\
\hline Mortality (\%) & $0(0)$ & $0(0)$ & NA \\
\hline Length of hospitalization [median (IQR)] & $12(9-22)$ & $19(12-24)$ & $0.033^{*}$ \\
\hline Still Hospitalized by day $28(\%)$ & $3(7.3)$ & $5(21.7)$ & 0.094 \\
\hline
\end{tabular}

Note: \# For those who had the symptoms initially, *Indicate statistically significant (P-value <0.05), NIV: Non-invasive ventilation; NA: Not Applicable; ICU: Intensive Care Unit; ARDS: Acute Respiratory Distress Syndromes; ECMO: Extracorporeal Membrane Oxygenation.

Patients who received combination therapy of DRV/COBI + RBV did not achieve faster resolution of fever or tachycardia compared to the DRV/COBI group (Table 2). Despite that the DRV/ $\mathrm{COBI}+\mathrm{RBV}$ group had longer duration of tachypnea than DRV/ $\mathrm{COBI}+\mathrm{RBV}$ group, which was not statistically significant $(P=0.063)$. Four patients $(17.4 \%)$ in DRV/COBI + RBV group were admitted to ICU, while no patient in DRV/COBI group required ICU admission during follow-up duration $(P<0.006)$. The median duration from the starting of treatment till ICU admission was 3 (1-3) days. Out of four patients requiring ICU admission, 3 patients required intubation and progressed to acute respiratory distress syndrome (ARDS), 2 patients required prone position, and none required ECMO support (Table 2). As patients in the DRV/COBI group had less complicated course, they had significantly shorter length of hospital stay than those in the DRV/COBI + RBV group (median 12 days vs. 19 days; difference -7 days; $P=0.033$ ). No mortality was reported by the end of our follow-up duration. The drop in hemoglobin of $\geq 2 \mathrm{~g} / \mathrm{dl}$ occurred significantly higher in the DRV/COBI + RBV group than in DRV/COBI group (34.8\% vs 7.9\%, $P=0.008$, respectively). The lowest hemoglobin during treatment duration in DRV/COBI + RBV group was $9.8 \pm 1.7$. Only 5 patients from both groups underwent full evaluation for the hemoglobin drop, of which 1 patient (in $\mathrm{DRV} / \mathrm{COBI}+\mathrm{RBV}$ group) met the criteria for hemolytic anemia (an increase in the bilirubin level to 1.5 times the baseline value, a haptoglobin level of $<0.38 \mathrm{~g} / \mathrm{L}$, or a reticulocyte count of $>110$ reticulocytes per 1000 erythrocytes) [14]. Elevated transaminase levels (defined as more than 3 folds of upper normal limit) were observed in $17.4 \%$ (4/23) patients in the DRV/COBI + RBV and $4.9 \%(2 / 41)$ patients in the DRV/COBI group. Out of those, one patient from each group required early discontinuation of DRV/ COBI because of a significant rise in transaminase levels. 


\section{Subgroup Analyses}

Twenty-eight patients were included in the subgroup analyses (patients with "early initiation" who started study treatment within 7 days of symptoms onset); 17 patients in DRV/COBI group and 11 patients in DRV/COBI + RBV group. Median duration from symptoms till starting therapy was 5 days (4-6) in both groups. No significant difference in demographic characteristics, baseline laboratory results, and clinical presentation/symptoms was found between the two groups. For the viral clearance, there was no significant difference between the two groups at the end of followup duration (day 28), nor at the other time points (i.e., day 7, 14 and 21 days). The mean duration till viral clearance was similar in both groups (15 days). However, 3 patients in the DRV/COBI + RBV required ICU admission, intubation, and progression to ARDS. The mean duration from starting DRV/COBI + RBV till ARDS was $4 \pm 1$ day.

\section{Risk Factors for Viral Clearance \& ARDS}

When assessing the association of variables to overall viral clearance, both Charlson's score and pre-existing myocardial infarction was found to be statistically significant in the univariate analysis. When assessed in the multivariable logistic regression, however, only Charlson's score was found to be inversely related to viral clearance. Only lymphopenia was found to be independently associated with increased risk of ARDS in multivariable logistic regression.

\section{DISCUSSION}

This retrospective study is the first study to explore the potential synergistic effect of ribavirin in combination with darunavir/cobicistat as treatment of COVID-19. Our results show that the combination was associated with faster viral clearance by day 7, 14 and 28 compared to darunavir/cobicistat alone, though the differences were not statistically significant.

Although previous in-vitro antiviral activity of darunavir showed no activity against SARS-CoV- 2 at clinically relevant concentrations [15], and one randomized controlled trial of 30 patients in China showed that DRV/COBI alone was not effective in the treatment of COVID-19 [13]; we thought of testing the clinical impact of co-administration of DRV/COBI with ribavirin as a possible strategy to augment darunavir's serum concentration and therefore a potential effective viral suppressing treatment for COVID-19. Both lopinavir and darunavir are within the same class and much similar in many aspects, however; darunavir is associated with less significant drug interactions making it preferable option in some patient/setting. As synergism was previously confirmed between lopinavir/ritonavir and ribavirin, we hypothesized that a similar synergism effect would be observed with darunavir and ribavirin, which can ultimately broaden treatment options during the pandemic. However, a high half maximal effective concentration of darunavir (EC50 $>100 \mu \mathrm{M}$ ) which found to be required for virus suppression in SARS-CoV-2 [15] could explain why synergism and concentration augmentation with ribavirin was not sufficient to reach clinically effective concentration/outcomes.

Systemic use of ribavirin (oral or intravenous) is known to cause reversible anemia due to hemolysis or bone marrow suppression [16]. Hemolytic anemia usually occurs after 10 days of therapy but may appear as early as 3 to 5 days after initiation of the drug [17]. A systematic review on oral ribavirin for the treatment of noninfluenza respiratory viral infections found that 11 studies reported hemolysis caused by ribavirin (14\%; 54/375 patients), ranging from mild to severe anemia requiring blood transfusions and ribavirin discontinuation [18]. In our study, a drop in hemoglobin of $\geq 2 \mathrm{~g} / \mathrm{dl}$ occurred in ribavirin group at around $35 \%$, which is higher than what was reported in previous systematic review [18]. Similarly, hemoglobin levels decreased to $<75 \%$ of the lower limit of normal was $26 \%$ in our study while it was reported to be $9 \%$ in another systematic review [17]. These differences in our results might be due to different dose, duration, comorbidities/conditions, or concurrent medications.

This study is the first of its kind to compare the outcomes of patients who received DRV/COBI \pm ribavirin for the treatment of COVID-19 infection. The two groups were much similar in their baseline characteristics and clinical presentation making the risk of biased results low. Also, we were able to compare both clinical, virological, and safety outcomes of both regimens. On the other hand, our study is limited by its retrospective observational design. However, it was the most feasible design given that our aim was to explore a new treatment strategy to guide whether testing it in a prospective interventional design is warranted. Small sample size was also one of the study's limitations which may affect the power of results. Previous studies have reported an association between viral clearance with both disease severity and mortality [19]. Therefore, viral load measurement of our sample could have provided an insight on baseline disease severity in our patients and its association with our study outcomes. Moreover, the viral load measurement could have been useful in identifying a subgroup of patients who might benefit more from the combination. Unfortunately, viral load measurement was not available during the study period.

\section{CONCLUSION}

In conclusion, the use of darunavir/cobicistat in combination with ribavirin was not associated with a faster viral clearance or better clinical outcomes compared to darunavir/cobicistat alone. Future prospective, randomized study with larger sample size is warranted to adequately evaluate the synergistic effect of the combined therapy.

\section{CONFLICT OF INTEREST}

The authors of this study have nothing to disclose concerning the possible financial or personal relationship with commercial entities that may have a direct or indirect interest in the subject matter of this study.

\section{REFERENCES}

1. Centers for Disease Control and Prevention (2020) 2019 novel coronavirus, wuhan, china.

2. (2020) World health organization's coronavirus disease (COVID-19).

3. Huang C, Wang Y, Li X, Ren L, Zhao J, et al. (2020) Clinical features of patients infected with 2019 novel coronavirus in wuhan, china. Lancet 395(10223): 497-506.

4. Chen N, Zhou M, Dong X, Qu J, Gong F, et al. (2020) Epidemiological and clinical characteristics of 99 cases of 2019 novel coronavirus pneumonia in wuhan, china: a descriptive study. Lancet 395(10223): 507-513.

5. Gorbalenya AE, Baker SC, Baric RS (2020) Severe acute respiratory syndrome-related coronavirus: The species and its viruses a statement of the coronavirus study group. bioRxiv.

6. Hsu LY, Lee CC, Green JA, Ang B, Paton NI, et al. (2003) Severe acute respiratory syndrome (SARS) in Singapore: clinical features of index patient and initial contacts. Emerg Infect Dis 9(6): 713-717. 
7. Chan KS, Lai ST, Chu CM, Tsui E, Tam CY, et al. (2003) Treatment of severe acute respiratory syndrome with lopinavir/ritonavir: a multicentre retrospective matched cohort study. Hong Kong Med J 9(6): 399-406.

8. WHO (2020) Landscape analysis of COVID therapeutics.

9. Chu CM, Cheng VC, Hung IF, Wong MM, Chan KH, et al. (2004) Role of lopinavir/ritonavir in the treatment of SARS: initial virological and clinical findings. Thorax 59(3): 252-256.

10. Liu F, Xu A, Zhang Y, Xuan W, Yan T, et al. (2020) Patients of COVID -19 may benefit from sustained Lopinavir-combined regimen and the increase of Eosinophil may predict the outcome of COVID -19 progression. Int J Infect Dis 95: 183-191.

11. Deng L, Li C, Zeng Q, Liu X, Li X, et al. (2020) Arbidol combined with LPV/r versus $\mathrm{LPV} / \mathrm{r}$ alone against corona virus disease 2019: A retrospective cohort study. J Infect 81(1): e1-e5.

12. Cao B, Wang Y, Wen D, Liu W, Wang J, et al. (2020) A Trial of lopinavirritonavir in adults hospitalized with severe covid-19. N Engl J Med 382(19): 1787-1799.

13. Jun Chen, Lu Xia, Li Liu (2020) Antiviral activity and safety of darunavir/ cobicistat for treatment of COVID -19. Open Forum Infectious Diseases $7(7)$.
14. Sandra R, Elizabeth J, Linda D, Larissa M (2003) Common adverse events associated with the use of ribavirin for severe acute respiratory syndrome in canada. Clin Infect Dis 37(8): 1139-1142.

15. De Meyer S, Bojkova D, Cinatl J, Van Damme E, Buyck C, et al. (2020) Lack of antiviral activity of darunavir against SARS-CoV-2. Int J Infect Dis 97: 7-10.

16. Gideon K, Susan K, Sandra K, Elizabeth P (2003) Ribavirin in the treatment of SARS: A new trick for an old drug? CMAJ 168(10): 12891292.

17. Chang CH, Chen KY, Lai MY, Chan KA (2002) Meta-analysis: ribavirininduced hemolytic anaemia in patients with chronic hepatitis C. Aliment Pharmacol Ther 16: 1623-1632.

18. Alan E, Michelle L (2015) Oral ribavirin for the treatment of noninfluenza respiratory viral infections: A systematic review. Ann Pharmacother 49(10): 1125-1135.

19. Elisabet P, Fayzan C, Russell M, Felix R, Shan Z, et al. (2020) SARS-CoV-2 viral load predicts COVID-19 mortality. Lancet Respir Med 8(9): E70. 